titelanzeige. Klickt man diesen Index an, kann man über den betreffenden Anfangsbuchstaben des fraglichen Begriffs an die betreffende Stelle in der „Hilfe“ gelangen.

\section{Ergebnisanzeige}

Je nach dem am Beginn der Recherche eingestellten „Anzeigemodus“ (chronologisch 0-9, chronologisch 9-0, alphabetisch) erhält man eine Kurztitelliste, die aufsteigend oder absteigend chronologisch oder alphabetisch geordnet ist. Durch Anklicken kann man sich einzelne Titel in Vollform oder als "Gesamtliste der Treffer" anzeigen bzw. durch Betätigen des Drucksymbols des Internetbrowsers ausdrucken lassen.

Das Hölderlin-Archiv bemüht sich, alle zu seiner Kenntnis gelangten Titel so rasch wie möglich auszuwerten und in die Datenbank einzubringen. Da neu eingegebene Titel sofort in der Datenbank zur Verfügung stehen, kann es später noch zu Modifikationen kom- men. Sie werden erst, wenn eine bestimmte Anzahl neuer Titel vorliegt, durchgeführt. Da Lücken in der Dokumentation der international erscheinenden Literatur zu Hölderlin nie zu vermeiden sind, sind wir für Hinweise stets dankbar!

\section{Fußnoten:}

1. Mit Erscheinen des Sonderbandes: Musikalien und Tonträger zu Hölderlin : 1806-1999. Stuttgart, 2000 wurde die gedruckte Internationale Hölderlin-Bibliographie eingestellt.

2. Die Programmierarbeiten wurden ausgeführt von Siegfried Eichmann (Host) und Torsten Johann (Internet) vom Statistischen Landesamt Baden-Württemberg, dem Host der IHB online.

3. Grundsätzliches dazu in: Sohnle, Werner Paul; Schütz, Marianne: Ein System für Hölderlin : die neue Hölderlin-Bibliographie: Was will und kann sie leisten? - In: HölderlinJahrbuch. - Stuttgart. - 27.1990/91.- S. 274295

Joachim Migl:

\title{
DFG-Projekt „Einbanddatenbank“ läuft an!
}

Im Januar 2001 hat die Deutsche Forschungsgemeinschaft die Förderung eines einbandkundlichen Projektes beschlossen, das die WLB gemeinsam mit der Herzog-August-Bibliothek Wolfenbüttel und der Staatsbibliothek zu Berlin / Preußischer Kulturbesitz beantragt hat. Hier sollen nun die Zielsetzung des Projektes und die sachlichen Hintergründe, hauptsächlich aber auch der Beitrag der WLB zu dem Projekt dargestellt werden.

Ist der individuell hergestellte, nach ganz persönlichen Vorlieben, handwerklichen Fertigkeiten und materiellen Möglichkeiten gestaltete, in jedem Falle aber unverwechselbare Bucheinband in heutiger Zeit die absolute Ausnahme, so war er in den Zeiten, bevor sich Massenproduktion und Verlegereinband durchsetzten, die Regel. Anfangs hatten „Buch“ und „Einband“ so wenig miteinander zu tun, dass fast immer der Kauf eines Buches nicht mehr bedeutete als die Erwerbung des Buchblocks, also der bedruckten bzw. beschriebenen Lagen, die vielleicht auch mal in einen Interimseinband geheftet sein konnten. Ob daraus einmal ein dauerhaft gebundenes Buch, und mit welchem Aufwand, Aussehen, Material der Einband geschaffen werden sollte, das blieb zunächst ganz allein die Angelegenheit des Eigentümers. Man wird 
sich leicht vorstellen können, dass die Neigung der Buchbesitzer, in eine Hülle für ihre Bücher zu investieren, zu allen Zeiten unterschiedlich groß war. Begnügten sich im 15. und 16. Jahrhundert die einen mit einem leichten und billigen Pappumschlag, der vielleicht unter Verwendung von älteren Papieren oder Pergamentstücken, z.B. aus aufgelösten Handschriften, gefertigt wurde, legten andere Wert auf schwere, lederbezogene Holzdeckel mit Beschlägen aus Metall. Wer 200 Jahre später seiner Bibliothek einen repräsentativen Charakter geben wollte, konnte seine Texte, die sonst z.B. in einem flexiblen Pergamenteinband steckten, in aufwendig verziertes und gefärbtes Leder einbinden lassen.

Welches Material jeweils besonders gerne benutzt wurde, welche Techniken und welche Verschönerungsmöglichkeiten gerade besonders gefragt waren, hat sich durch die Zeiten zwar immer wieder geändert und war allgemeinen stilgeschichtlichen bzw. modischen Entwicklungen unterworfen. Aber allen Einbänden war gemeinsam, dass sie ausnahmslos in Handarbeit angefertigt wurden. Die von den Bucheigentümern beauftragten Buchbinder und Werkstätten hinterließen dabei manchmal unverwechselbare Merkmale eines ganz persönlichen Arbeitsstils und benutzten vielfach eindeutig identifizierbare Werkzeuge, so dass die Kenntnis solcher Charakteristika die Zuweisung eines ansonsten nicht signierten Einbandes an einen Binder bzw. eine Werkstatt erlaubt! Denkt man nun an die häufig sehr wechselhaften und abenteuerlichen Geschichten, die Bücher mitsamt ihren Einbänden im Laufe eines jahrhundertelangen Lebens hinter sich bringen können, dann wird klar, wie die Einbandkunde für die Buchgeschichte nutzbar gemacht werden kann
Wenn in Erfahrung zu bringen ist, wer zu welcher Zeit und an welchem Ort einem konkreten Buchblock ein bis heute überliefertes Gewand verpasst hat, dann gibt das in Kombination mit eventuellen Besitzeinträgen, Marginalien oder sonstigen individuellen Spuren im Buch wertvolle Hinweise auf seine Geschichte, seine früheren Besitzer und Aufbewahrungsorte.

Natürlich hat sich die Buchforschung diese Zusammenhänge für ihre Fragen schon immer zunutze gemacht und eine fast unüberschaubare Literaturliste mit wichtigen Beiträgen zu einzelnen Buchbindern, ihren Werkstätten oder besonders interessanten „Buchgeschichten" hervorgebracht. So gesehen ist der Aufbau einer Einbanddatenbank keine methodische Revolution. Doch die Ausgangslage schreit - in zweifacher Hinsicht - förmlich nach einer Datenbanklösung.

Da ist erstens die unübersehbar große Datenmenge. Unendlich viele kleine, aber charakteristische und deshalb wichtige Details der Einbandherstellung und -verzierung müssen erfasst und beschrieben werden.

Und zweitens kann die angestrebte Übersicht angesichts der aktuellen Besitzsituation nur in einer virtuellen Einbandsammlung erreicht werden. Unendlich viele historische Einbände stecken heute in zahlreichen Bibliotheken und Sammlungen, die wiederum Reste und Splitter früherer Bibliotheken und Sammlungen aufbewahren, in denen noch ältere Bibliotheken und Sammlungen aufgegangen sind. Die Eigentumsverhältnisse überlagern sich mehrfach, die ursprünglichen Zusammengehörigkeiten sind meistens nur noch mühsam erkennbar. Die Zusammenführung und der Vergleich ähnlicher oder identischer Einbandmerk- 


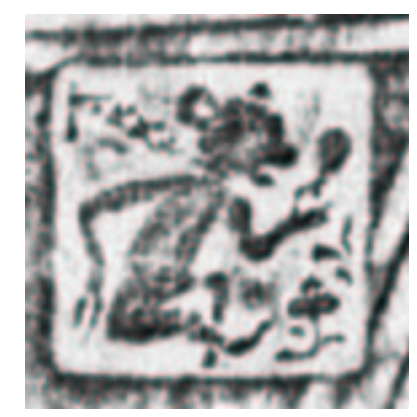

Stempel mit Motiv Affe, nach dem die Werkstatt benannt ist. male ist anders als in einer Datenbank kaum möglich.

Obwohl das geplante Projekt grundsätzlich für die Erfassung von Einbänden aller Epochen und Zeiten offen sein will, beginnen die drei Partner mit einem verhältnismäßig kleinen Zeitsegment, dem späteren 15 . und früheren 16. Jahrhundert. Das hat einen theoretischen und einen damit zusammenhängenden, sehr praktischen Grund.

Zum einen entsprechen die meisten verzierten Einbände dieser Zeit einem bestimmten Typus. Sie gehören zu den sogenannten blindgeprägten Einbänden, bei denen auf einem lederbezogenen Buchdeckel mit verschiedenen Prägewerkzeugen reliefartige Abdrucke erzeugt werden, wobei anfangs ganz überwiegend Einzelstempel, dann zunehmend auch Rollen und größere Platten benutzt wurden. Wenngleich diese Verzierungstechnik auch in späterer Zeit immer noch angewandt wurde und selbst bei Handeinbänden des 20. Jahrhunderts noch eingesetzt wird, sind die blindgeprägten Einbände der Jahrzehnte vor und nach 1500 doch eine besondere Gruppe, da die benutzten Werkzeuge ganz überwiegend Einzelanfertigungen sind und ihre Besitzer nicht allzu oft wechseln, damit also mit großer Sicherheit auf Binder und Werkstatt schließen lassen.

Zum anderen erfreuen sich ebendiese Werkzeuge gerade wegen ihres individuellen Aussehens schon bei mehreren Generationen von Einbandforschern besonderer Aufmerksamkeit. Wir bearbeiten somit kein gänzlich unerschlossenes Gebiet, sondern stützen uns auf viele Vorarbeiten namhafter Historiker, die uns ihre Arbeitsunterlagen z.T. hinterlassen haben. Und damit kommen wir nun zu der Frage, weshalb ausgerechnet die Landesbibliothek einen Bei- trag zur geplanten Datenbank liefern kann.

Die WLB besitzt seit vielen Jahrzehnten die Arbeitsmaterialien eines der wichtigsten deutschen Einbandforscher des 20. Jahrhunderts, das komplette Archiv zu spätgotischer Einbandverzierung von Ernst Kyriss. Wie sieht dieses Material aus?

Das bis heute übliche Verfahren, die Motive auf den Einbänden auf Papier zu übertragen, ist die Durchreibung. Auf ein möglichst glatt gestrichenes $\mathrm{Pa}$ pier wird mit flach angesetztem Bleistift das Relief so abgepaust, wie das wohl jeder auch mal mit Geldmünzen gemacht hat. Der Effekt ist derselbe: Die Konturen, die Grenzen zwischen vertieften und erhabenen Partien werden auf dem Papier sichtbar. Eine dreidimensionale Stempelprägung wird zweidimensional abgebildet. Wie gut das jeweils funktioniert, hängt nicht nur von der Qualität der Vorlage ab - viele Einbände sind so stark berieben, dass das Profil der Prägungen längst weg ist - , vor allem die Erfahrung und das Geschick des "Durchreibers" wirken sich auf das Ergebnis aus. Man kann sicher behaupten, dass die Durchreibungen von Ernst Kyriss zu dem Besten gehören, was mit dieser Methode hergestellt worden ist. Das Verfahren mag zunächst belächelt werden, aber wer sich ernsthaft überlegt, wie man schnell und in guter Qualität exakte Abbildungen der geprägten Reliefs herstellen kann, wird bald feststellen, dass die Methode praktisch konkurrenzlos ist. Wirklich schwierig und längst nicht immer befriedigend sind Fotografien, für die eine sehr komplizierte Ausleuchtung notwendig ist, um unerwünschten Schattenwurf zu verhindern. Fotos sind auBerdem teuer und führen nicht automatisch zu einer wirklichkeitsgetreuen 
Größenwiedergabe. Am Rande sei darauf hingewiesen, dass ähnliche Methoden auch in anderen wissenschaftlichen Zusammenhängen angewandt werden, wenn es z.B. um die Kopie von Inschriften oder sonstigen reliefartigen Artefakten geht. Dort benutzt man sogenannte Abklatsche, eine Art Gipsabguss. Diese machen oft Informationen auf der Vorlage erkennbar, die auf Zeichnungen und Fotos fehlen.

Ist es sinnvoll und wissenschaftlich vertretbar, nun in der Datenbank nur die Kopien zu erfassen? Müsste man nicht doch direkt auf die Originalvorlagen zurückgreifen? Drei gute Gründe sprechen bei dieser Frage für die Beschäftigung mit den Kopien:

Die Durchreibungen auf Papier sind leichter in elektronische Bilder zu überführen als die Vorlagen. Da es sich um Schwarz-Weiß-Vorlagen handelt, gibt es beim Scannen keine Komplikationen, während Direktscans von Einbänden schwierig sind.

Passionierte Einbandforscher wie Ernst Kyriss haben einen großen Teil ihrer Arbeitszeit mit nichts anderem verbracht, als Einbandverzierungen zu sammeln und zu ordnen. Diese Arbeitsleistung ist heute von Einzelnen kaum noch zu erbringen, und selbst wenn, dann wäre es die Wiederholung einer Arbeit, die ein anderer doch schon gemacht hat. Außerdem haben die Durchreibungssammlungen aus der Zeit vor dem 2. Weltkrieg den Vorteil, noch Material zu enthalten, das heute untergegangen oder verschollen ist.

Und schließlich haben uns Forscher wie Ernst Kyriss schon sehr viel Arbeit abgenommen. Deren Interesse war ja dasselbe wie unser heutiges, sie haben die Durchreibungen keineswegs als Selbstzweck angesehen, sondern als
Basis für darauf aufbauende Schlussfolgerungen. In diesem Sinne haben sie ihr Material schon sortiert und strukturiert. Da steckt enorm viel Arbeit und Wissen dahinter, das zu übergehen geradezu sträflich wäre.

Ernst Kyriss war eigentlich Bauingenieur und begann erst nach seiner Frühpensionierung 1924 - er war gerade 43 Jahre alt - sich für historische Bucheinbände zu interessieren. 1929 begann er mit dem planmäßigen Durchreiben von spätgotischen Einbänden. Die damit begründete Sammlung führte er bis wenige Jahre vor seinem Tod 1974 fort. In den rund 40 Jahren, die er sich mit dieser Materie beschäftigte, besuchte er über 60 Bibliotheken, registrierte mehr als 50.000 Einbände und hielt auf schätzungsweise mehr als 20.000 Blättern Einbandverzierungen mittels Durchreibungen fest. Aber Kyriss hatte nicht nur unglaublich viel Zeit für seine Leidenschaft, er besaß zudem einen schon fast erschreckend perfekt ausgebildeten Ordnungssinn, der ihn in die Lage versetzte, die ungeheure Materialflut mit den Möglichkeiten seiner Zeit zu ordnen und die enthaltenen Zusammenhänge zu entdecken. Einen kleinen Teil seiner Arbeitsergebnisse publizierte er in den 50 er Jahren in dem bis heute unverzichtbaren Standardwerk „Verzierte gotische Einbände im alten deutschen Sprachgebiet“. In den Arbeitsmappen von Kyriss steckt weit mehr an Information, als es in diesem Werk jemals hätte veröffentlicht werden können. Mit dem DFG-Projekt wollen wir auch dieses Wissen zugänglich machen. Dafür ist von entscheidender Bedeutung, dass das Ablagesystem und die Arbeitsweise von Ernst Kyriss rekonstruiert werden können. Denn nur daraus, sozusagen in der Fortsetzung

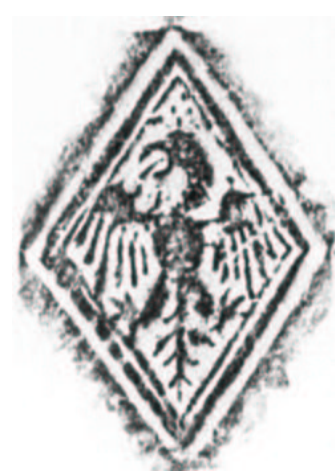

Rhombischer Stempel mit einem heraldisch dargestellten Adler

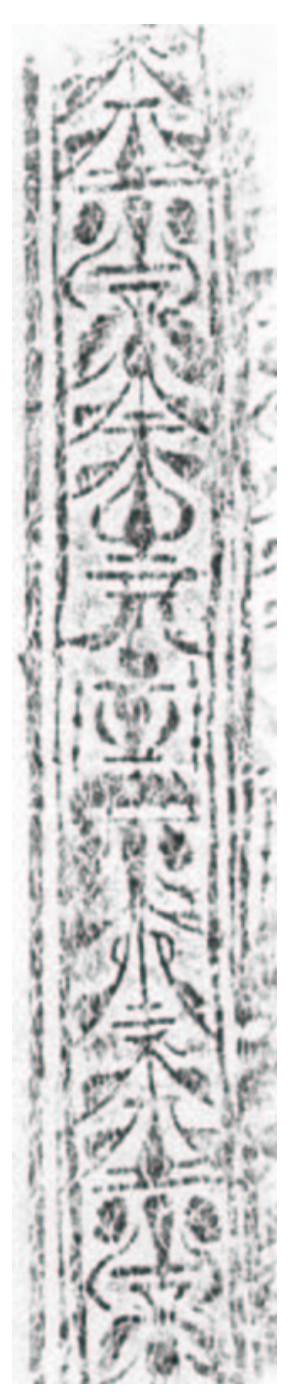

Abdruck einer Rolle 


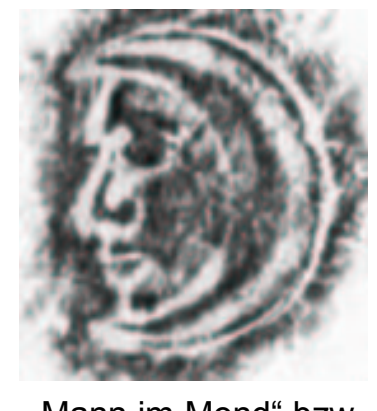

„Mann im Mond“ bzw. nach Thesaurusliste: Mond mit Gesicht

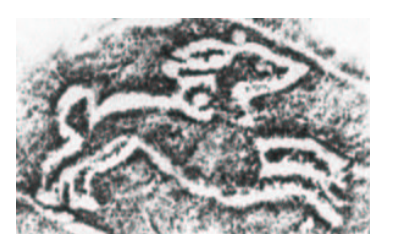

Stempel mit dem Motiv Hase seiner Systematik, erschließt sich für uns heute der Zugang zu der großen Datenflut. Als die Landesbibliothek außer der Einbandsammlung von Ernst Kyriss und seiner Handbibliothek auch sein Durchreibungsarchiv erworben hatte und die Übergabe erfolgte, wanderten rund 350 Jurismappen in unsere Magazine, wo sie exakt in der übernommenen Ordnung aufgestellt wurden. Den Hauptteil des Materials machen die alphabetisch nach Durchreibeorten sortierten Mappen aus, in denen Kyriss die in den besuchten Bibliotheken angefertigten Durchreibungen sammelte. Von ungleich größerer Bedeutung für uns sind die dazu geführten Registerbücher und die darauf aufsetzenden Kumulationsstufen. Die Zusammenfassung von einzelnen Stempeln zu Gruppen, die wiederum häufig identisch mit Werkstätten sind, konnte erst parallel zur ständigen Verbreiterung der Materialbasis erfolgen. Erst in der Zusammenführung von Stempelfunden aus verschiedenen Fundorten rundete sich allmählich das Bild ab: Langsam konnte so die Zusammenstellung aller von einer Werkstatt benutzen Werkzeuge immer vollständiger werden. Um überhaupt so weit zu kommen, ließ sich verständlicherweise hin und wieder Redundanz nicht vermeiden: In den Sammlungen fanden sich natürlich auch viele Bücher, die identische Verzierungen aufwiesen, ohne dass Kyriss dies sofort auffiel. So konnte es z.B. vorkommen, dass Kyriss in mehreren Bibliotheken insgesamt vielleicht 50 oder 60 Stempel durchrieb, bis inm deren Zusammengehörigkeit unumstößlich bewiesen schien und nach der Tilgung der vorsichtshalber mitkopierten Durchreibungsdubletten oder sogar -tripletten am Ende noch ein Rest von 20 Stempeln übrig blieb, der dann als Gruppe feststand. Manchmal fand Kyriss noch viele Jahre später in weiteren Orten noch einen 21. oder 22. Stempel. Aus all dem erhellt, dass es nicht sinnvoll wäre, das Gesamtmaterial in die Datenbank einzubringen, da es geradezu verhängnisvoll wäre, von ein und demselben Stempel mehrere Durchreibungen festzuhalten. Es sind vielmehr die Gruppenzusammenstellungen, also die Reduktion des Materials mit dem Ziel, jeden Stempel aus jeder Werkstatt genau einmal erfasst zu haben, die für das Projekt von Bedeutung sind. Erst bei dem Nachweis, welcher Stempel auf welchen Einbänden in welchen Bibliotheken vorkommt, ist der Rückgriff auf das Gesamtmaterial wieder unumgänglich.

Ausgehend von diesen Überlegungen haben wir im Frühjahr die Blätter, auf denen Kyriss seinen Kenntnisstand über den Stempelvorrat einzelner Werkstätten zusammengefasst hatte, zur Verfilmung und Digitalisierung an die Firma Hermann und Krämer nach Garmisch-Partenkirchen gegeben. Diese Methode, zuerst zu verfilmen und vom Film dann digitale Kopien herzustellen, ist nicht nur die preiswertere Variante im Vergleich zur Direktdigitalisierung, sie zeitigt darüber hinaus sehr gute Ergebnisse, wie Tests im Vorfeld gezeigt haben, und trägt mit der Anfertigung eines haltbaren Filmes außerdem auch noch zur langfristigen Materialsicherung bei. Rund 1700 Blätter mit schätzungsweise 20.000 Stempeln liegen jetzt auf Film und digital auf CD-ROM vor. Damit steht das Bildmaterial in großem Umfang bereit; es liegt aber auf der Hand, dass damit allein noch keine Datenbank aufgebaut werden kann. Die entsteht vielmehr erst dadurch, dass die Stempel, Rollen und Platten selbst in festgelegten Kategorienschemata beschrieben, und die mannigfachen Beziehungen der Werkzeuge zur Werkstatt bzw. zu den buchbinderischen Einheiten durch Verknüp- 


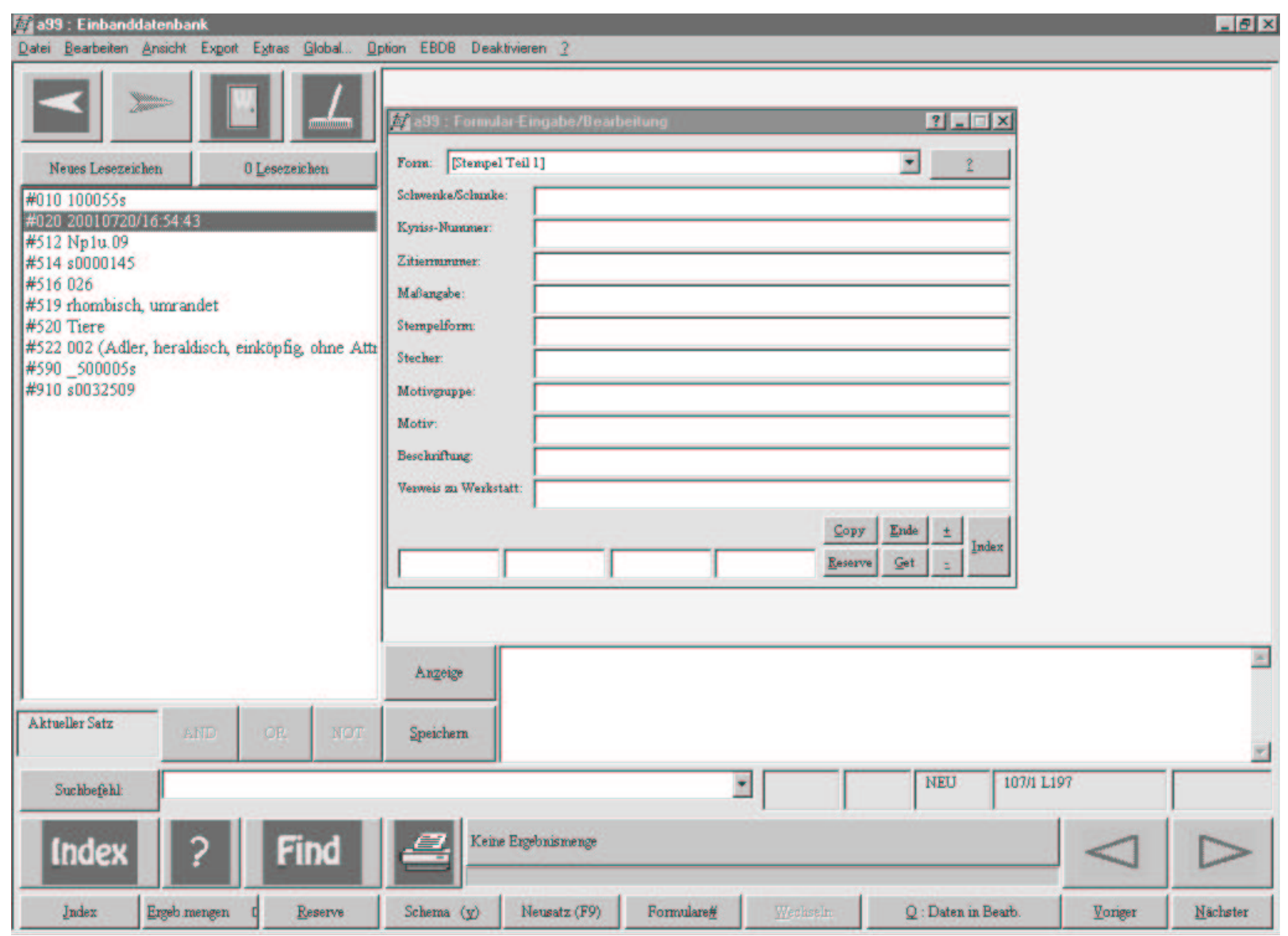

fungen von Datensätzen abgebildet werden. Das soll mit Hilfe des Datenbankprogramms Allegro bewerkstelligt werden, das zu diesem Zweck anzuschaffen und zu konfigurieren war letzteres ein großes Verdienst der Kollegen in Berlin. Das Gesamtmodell musste und muss selbstverständlich mit den Projektpartnern diskutiert werden, die ja ebenfalls ihre Daten in die gemeinsame Datenbank einspeisen wollen. Bedenkt man die zahlreichen Details, die in der Planung einer solchen Lösung berücksichtigt sein wollen, die Vielzahl von Sonderwünschen, die für alle gültigen Konventionen, die Standards und Arbeitsverfahren, die bis zu einem gewissen Grad vereinheitlicht werden müssen, dann wird leicht verständlich, dass der Aufbau einer kombinierten Text-Bild-Datenbank dieser Art einen erheblichen zeitlichen Vorlauf mit zahlreichen Besprechungen voraussetzt.
Es wird mehrere Typen von Datensätzen geben, die untereinander verknüpft werden können. Diese Satztypen sind jetzt festgelegt für:

Werkzeuge

Werkstätten

Buchbinderische Einheiten

Titel

Besitzer- und Vorbesitzer.

Der eigentliche Kern des Projektes ist die Dokumentation der Zusammenhänge von Werkzeugen, Werkstätten und buchbinderischen Einheiten. Vorsicht ist vor allem bei Titelsätzen geboten, da es nicht Sinn der Datenbank sein kann bzw. darf, reine Kataloginformationen zu wiederholen. Genauso wenig soll die Einbanddatenbank gleichzeitig ein Instrument von vollständigen Provenienznachweisen werden. Hier gilt die Regel, dass festgehalten werden soll, was an
Allegro-Oberfläche mit Erfassungsmaske für einen Einbandstempel. 
Information zur Hand ist, weitere Recherchen aber nicht durchgeführt werden sollen. Lösbar scheint inzwischen das Problem der Terminologie, also der normierten Benennung von Motiven und Stempeltypen, da eine Arbeitsgruppe des AEB (Arbeitskreis für die Erfassung und Erschließung historischer Bucheinbände) gerade eine Liste speziell zu diesem Thema entwickelt. Vieles ist noch $\mathrm{zu}$ regeln oder auch im Lauf des Projektes noch zu klären. Glücklicherweise ist bei nur drei Partnern zu Beginn einiges schnell und direkt am Telefon zu besprechen.

Seit Mitte April hat sich in der WLB Herr Giertz in die Materie eingearbeitet. Seine Aufgabe besteht darin, aus dem Bildrohmaterial datenbankverträgliche

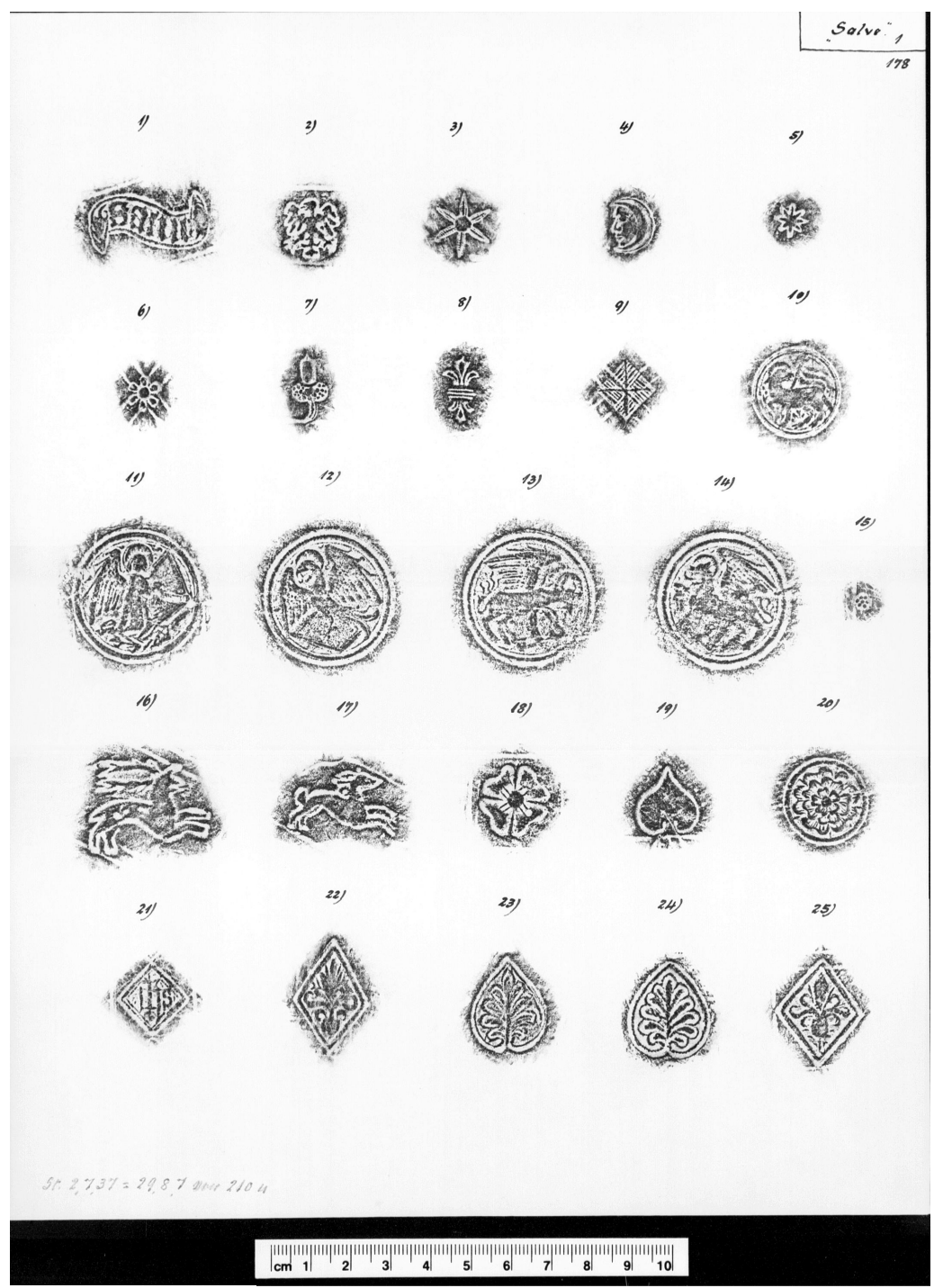

WLB-forum 3. Jg. 2001, Heft 2
Häppchen zuzubereiten und die anfallenden Beschreibungskategorien auszufüllen. Außerdem wird die inhaltliche Redaktion für das Gesamtprojekt in Stuttgart liegen. Die Aufarbeitung des Kyriss-Materials verlangt zudem nicht selten fast kriminalistische Fähigkeiten, kommt es doch häufig darauf an, den Weg (und die vielen Neben- und Holzwege) der Erkenntnis dieses Einbandforschers aus seiner Hinterlassenschaft zu rekonstruieren, ggf. auch auf der Basis des aktuellen Wissens zu modifizieren, interne Widersprüche und Ungereimtheiten aufzuklären usw.

Sobald es eine Suchoberfläche geben wird, mit der Interessenten die Datenbank möglichst differenziert aber doch komfortabel befragen können, wird sich sicherlich der Nutzen dieser großen Mühe zeigen.

Beispiel für eine Gruppe von Durchreibungen der Stempel einer Werkstatt („Salve 1“, interne Nr. 178) aus dem Archiv von Ernst Kyriss, mit Maßstab verfilmt und digitalisiert. 